\title{
Article \\ Solvent-Free C-3 Coupling of Azaindoles with Cyclic Imines
}

\author{
Khadija Belasri ${ }^{1,2}$, Ferenc Fülöp ${ }^{1,2}$ and István Szatmári ${ }^{1,2, *}$ \\ 1 Institute of Pharmaceutical Chemistry and MTA-SZTE Stereochemistry Research Group, University of \\ Szeged, H-6720 Szeged, Hungary; Khadija.belasri@pharm.u-szeged.hu (K.B.); \\ fulop@pharm.u-szeged.hu (F.F.) \\ 2 Institute of Pharmaceutical Chemistry, Interdisciplinary Centre of Excellence, University of Szeged, \\ H-6720 Szeged, Hungary \\ * Correspondence: szatmari.istvan@pharm.u-szeged.hu
}

Academic Editor: Antal Csámpai

Received: 3 September 2019; Accepted: 2 October 2019; Published: 4 October 2019

\begin{abstract}
By direct coupling 7-azaindole and cyclic imines, such as 3,4-dihydroisoquinoline, 6,7-dihydrothieno[3,2-c]pyridine, 3,4-dihydro- $\beta$-carboline, and 4,5-dihydro-3H-benz[c]azepine, new 3-substituted 7-azaindole derivatives have been synthesized. The reaction was extended to 4-azaindoles and 6-azaindoles, as electron-rich aromatic compounds. The lowest reactivity was observed in the case of C-3 substitution of 5-azaindole. In this case, the aza-Friedel-Crafts reaction took place by using $10 \mathrm{~mol} \%$ of $p$-toluenesulfonic acid ( $p$-TSA) as the catalyst. The role of the acid catalyst can be explained by the different $\mathrm{pKa}$ values of the azaindoles. All reactions were performed in solvent-free conditions by using both classical heating and microwave irradiation. In all cases, microwave heating proved to be more convenient to synthesize new C-3-substituted azaindole derivatives.
\end{abstract}

Keywords: cyclic imines; 7-azaindole; 6-azaindole; 4-azaindole; 5-azaindole; aza-Friedel-Crafts reaction; microwave reaction

\section{Introduction}

7-Azaindole is a well-known hinge-binding element in kinase inhibition [1-5]. The $\mathrm{N}$ atom of the pyridine ring and the $\mathrm{NH}$ group of the pyrrole moiety of 7-azaindole serve as the hydrogen bond acceptor and donor, respectively, to make bidentate hydrogen bonds with the hinge region of the kinase. 7-Azaindole has five modification sites where various substituents can be readily attached to obtain 7-azaindole derivatives with improved activity. Among them, vemurafenib [6], a B-RAF kinase (serine -threonine kinase (STK)) inhibitor, is the first U.S. Food and Drug Administration (FDA)-approved, 7-azaindole-based kinase drug for the treatment of melanoma [7]. Vemurafenib was discovered through lead optimization, starting from a small 7-azaindole fragment, and now it is recognized as the first successful example of a "fragment-based" drug discovery approach [8]. Some derivatives have been developed that target various kinds of kinases, including Janus kinase 3 (JAK3; a cytoplasmic tyrosine kinase (TK)) [9]; colony stimulating factor 1 receptor (CSF1R; a TK receptor) [10]; aurora kinases (STKs) [11]; and Rho-associated, coiled coil-containing protein kinase 1 (ROCK1; STK) [12].

Thanks to the close similarity of azaindoles to the indole skeleton, C-3 functionalization of these compounds has been postulated $[13,14]$. Most of the methods known already for the synthesis of 3-functionalized 7-azaindole derivatives have applied multistep transformations. Particular efforts have been made to insert another biologically active moiety, such as tetrahydroisoquinoline, into position three. In this case, the synthesis of 1-(7-azaindol-3-yl)-1,2,3,4-tetrahydroisoquinoline involves the coupling of 7-azaindole with $\mathrm{N}$-protected tetrahydroisoquinoline under iron and copper catalysis $[15,16]$. 
The catalyst-free direct coupling of partially-saturated cyclic amines and indole as an electron-rich aromatic compound, via a modified aza-Friedel-Crafts reaction, has recently been published by our research group $[17,18]$. The reaction has been extended by using indole-2-carboxylic acid as a substrate, leading to the formation of $\gamma$-amino acid derivatives [17].

Our aim was to perform a systematic study of the synthesis of azaindole derivatives (7-, $4-, 5-$, and 6-azaindoles), applying the modified aza-Friedel-Crafts reaction using cyclic imine substrates, such as 3,4-dihydroisoquinoline; 6,7-dihydrothieno[3,2-c]pyridine; 3,4-dihydro- $\beta$-carboline; and 4,5-dihydro-3H-benz[c]azepine.

\section{Results}

In our initial experiments, 7-azaindole (compound 1) was reacted with 1.5 equivalent of 3,4-dihydroisoquinoline (compound 2), which was synthesized using a method from the literature (Scheme 1) [18]. The reaction between compounds $\mathbf{1}$ and $\mathbf{2}$ was performed in solvent-free conditions by classical heating (oil bath) at $60^{\circ} \mathrm{C}$ (i). Based on thin-layer chromatography (TLC), the reaction over a time of $18 \mathrm{~h}$ resulted in the formation of a multi-spot reaction mixture; the desired product (compound 3) was isolated by chromatography, with a yield of $31 \%$. Since the yield was not satisfactory, the reaction was repeated at $80^{\circ} \mathrm{C}$ (ii). A $10 \mathrm{~h}$ reaction led to the formation of compound 3, with a yield of $49 \%$. Despite increasing the temperature further $\left(100^{\circ} \mathrm{C}\right.$, (iii)), the relatively long reaction led to compound 3 only with a poor yield $(28 \%)$. When the reaction was repeated under microwave irradiation by testing three different reaction conditions (Table 1), 3-(1,2,3,4-tetrahydroisoquinolin-1-yl)-7-azaindole (3) was isolated with a yield of $81 \%$ after $120 \mathrm{~min}$ at $100{ }^{\circ} \mathrm{C}$ (Table 1). It is interesting to note that for microwave reactions, 2 equivalents of 3,4-dihydroisoquinoline was applied to provide homogeneity for the reaction mixture.

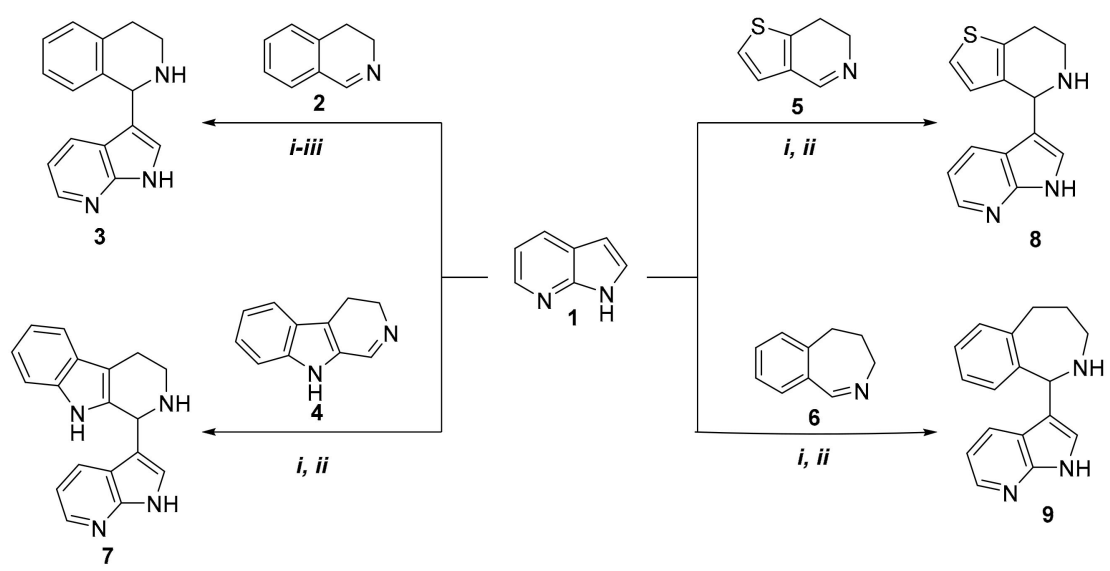

Scheme 1. Synthesis of 7-azaindole derivatives, starting from different cyclic imines.

Table 1. Reaction conditions for the synthesis of the azaindole compounds 3 and 7-9.

\begin{tabular}{|c|c|c|c|c|c|c|}
\hline Products & Reaction Time $^{a}$ & Temperature $^{a}$ & Yield a (\%) & Reaction Time $^{b}$ & Temperature $^{b}$ & Yield $^{b}(\%)$ \\
\hline \multirow{3}{*}{3} & $18 \mathrm{~h}$ & $60^{\circ} \mathrm{C}(i)$ & 31 & $2 \mathrm{~h}$ & $80^{\circ} \mathrm{C}(\mathrm{ii})$ & 55 \\
\hline & $10 \mathrm{~h}$ & $80^{\circ} \mathrm{C}(i i)$ & 49 & $4 \mathrm{~h}$ & $80^{\circ} \mathrm{C}(i i)$ & 63 \\
\hline & $6 \mathrm{~h}$ & $100{ }^{\circ} \mathrm{C}(\mathrm{iii})$ & 28 & $2 \mathrm{~h}$ & $100^{\circ} \mathrm{C}(\mathrm{iii})$ & 81 \\
\hline 7 & $10 \mathrm{~h}$ & $80^{\circ} \mathrm{C}(\mathrm{ii})$ & 56 & $2.5 \mathrm{~h}$ & $100^{\circ} \mathrm{C}(\mathrm{iii})$ & 75 \\
\hline 8 & $6 \mathrm{~h}$ & $80^{\circ} \mathrm{C}(i i)$ & 76 & $2 \mathrm{~h}$ & $100{ }^{\circ} \mathrm{C}(\mathrm{iii})$ & 89 \\
\hline 9 & $20 \mathrm{~h}$ & $80^{\circ} \mathrm{C}(\mathrm{ii})$ & 63 & $3 \mathrm{~h}$ & $100^{\circ} \mathrm{C}(\mathrm{iii})$ & 78 \\
\hline
\end{tabular}

${ }^{\mathrm{a}}$ classical heating, ${ }^{\mathrm{b}}$ microwave irradiation.

The reaction was extended by using other cyclic imines, such as 3,4-dihydro- $\beta$-carboline (compound 4) [19]; 6,7-dihydrothieno[3,2-c]pyridine (compound 5) [20]; and 4,5-dihydro-3Hbenz[c]azepine (compound 6) [21,22]. Reactions were performed by using both oil-bath heating 
and microwave irradiation (Scheme 1). Table 1 shows that the corresponding products-compounds 7, 8, and 9-were isolated in higher yields when microwave conditions were applied. It can be concluded that 6,7-dihydrothieno[3,2-c]pyridine (5) was found to be the most reactive cyclic imine. Yields and the applied reaction conditions are summarized in Table 1.

With the optimal reaction conditions in hand, we focused on extending the series of electron-rich aromatic compounds in a modified aza-Friedel-Crafts reaction. Accordingly, 4-azaindole (compound 10) and 6-azaindole (compound 11) were also reacted with cyclic imines 2, 4, 5, and 6 (Scheme 2).
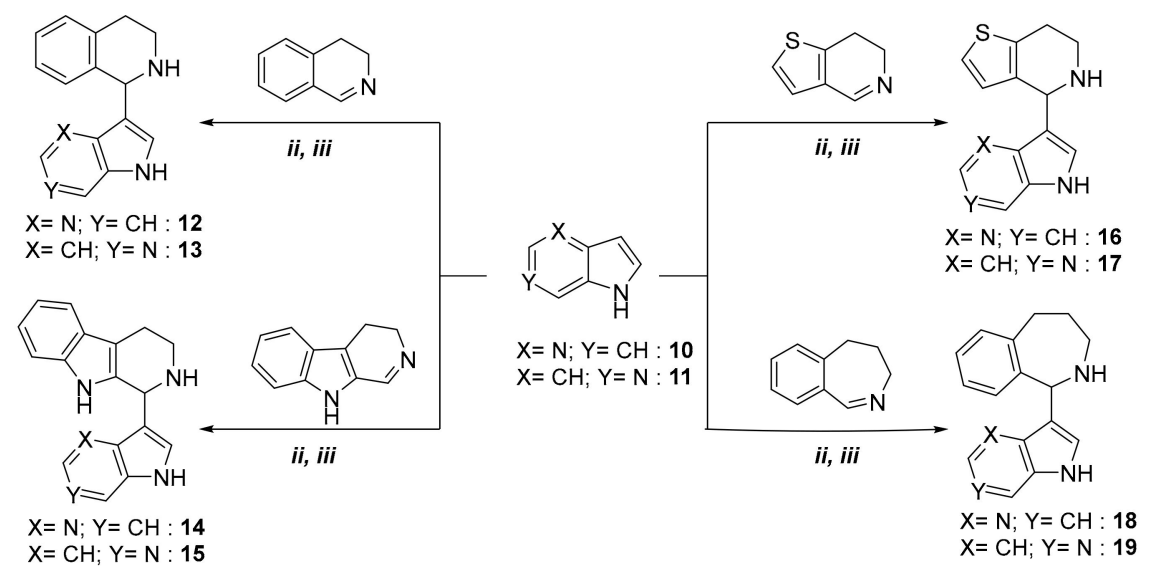

Scheme 2. The extension of a modified aza-Friedel-Crafts reaction, starting from 4-azaindole and 6-azaindole.

Reactions were performed under solvent-free conditions by both classical heating and using microwave irradiation. The new azaindole derivatives, formed in reaction times indicated in Table 2, were isolated and purified by crystallization or column chromatography (see Material and Methods). It can be concluded that the use of microwave irradiation afforded the desired products (compounds 12-19) in higher yields and with significantly shorter reactions, compared with those using oil-bath heating (Table 2).

Table 2. Reaction conditions for the synthesis of the azaindoles 12-19.

\begin{tabular}{|c|c|c|c|c|c|c|}
\hline Products & Reaction Time $^{a}$ & Temperature $^{a}$ & Yield $^{\text {a }}(\%)$ & Reaction Time $^{b}$ & Temperature $^{b}$ & Yield $^{b}(\%)$ \\
\hline 12 & $20 \mathrm{~h}$ & $80^{\circ} \mathrm{C}(i i)$ & 49 & $2.5 \mathrm{~h}$ & $100{ }^{\circ} \mathrm{C}($ iii $)$ & 70 \\
\hline 13 & $22 \mathrm{~h}$ & $80^{\circ} \mathrm{C}(i i)$ & 39 & $2.5 \mathrm{~h}$ & $100^{\circ} \mathrm{C}(i i i)$ & 65 \\
\hline 14 & $16 \mathrm{~h}$ & $80^{\circ} \mathrm{C}$ (ii) & 52 & $2.5 \mathrm{~h}$ & $100^{\circ} \mathrm{C}(i i i)$ & 69 \\
\hline 15 & $20 \mathrm{~h}$ & $80^{\circ} \mathrm{C}(i i)$ & 48 & $2.5 \mathrm{~h}$ & $100^{\circ} \mathrm{C}(\mathrm{iii})$ & 62 \\
\hline 16 & $7 \mathrm{~h}$ & $80^{\circ} \mathrm{C}$ (ii) & 63 & $2.5 \mathrm{~h}$ & $100^{\circ} \mathrm{C}$ (iii) & 85 \\
\hline 17 & $8 \mathrm{~h}$ & $80^{\circ} \mathrm{C}$ (ii) & 62 & $2.5 \mathrm{~h}$ & $100^{\circ} \mathrm{C}(\mathrm{iii})$ & 75 \\
\hline 18 & $21 \mathrm{~h}$ & $80^{\circ} \mathrm{C}(i i)$ & 57 & $3 \mathrm{~h}$ & $100^{\circ} \mathrm{C}(i i)$ & 68 \\
\hline 19 & $25 \mathrm{~h}$ & $80^{\circ} \mathrm{C}(i i)$ & 55 & $3 \mathrm{~h}$ & $100^{\circ} \mathrm{C}(i i i)$ & 64 \\
\hline
\end{tabular}

Since the catalyst-free coupling of 7-, 6-, and 4-azaindoles resulted in the desired C-3 aminoalkylated derivatives, we focused our attention on the aza-Friedel-Crafts reaction of 5-azaindole (compound 20). In our first experiment, 20 was reacted with 3,4-dihydroisoquinoline (2) as representative cyclic imine (Scheme 3). However, even when applying different reaction conditions (classical heating, microwave irradiation) and different temperatures $\left(80^{\circ} \mathrm{C}\right.$ (ii), $100{ }^{\circ} \mathrm{C}$ (iii), $120^{\circ} \mathrm{C}($ iv) )), target compound 21 did not form. There was no conversion at lower temperature $\left(80^{\circ} \mathrm{C}, 100^{\circ} \mathrm{C}\right)$, while higher temperature $\left(120^{\circ} \mathrm{C}\right)$ resulted in the formation of a multi-spot reaction mixture. Since $p$-toluenesulfonic acid ( $p$-TSA) is a frequently applied acid catalyst in the modified three-component Mannich reaction [23-25], we decided to examine its effect on the reaction between 20 and 3,4-dihydroisoquinoline. First, 
10 mol \% of $p$-TSA was tested by using oil-bath conditions. In this case, TLC showed the formation of a new compound, which after isolation (30\%) proved to be the desired 5-azaindole derivative 21. Since the reaction needed a relatively long reaction time $(19 \mathrm{~h})$ and resulted in a poor yield, it was repeated by using microwave irradiation. In this case, $100{ }^{\circ} \mathrm{C}$ proved to be optimal, and 3-(1,2,3,4-tetrahydroisoquinolin-1-yl)-5-azaindole (21) was isolated with a yield of 72\% (Table 3).

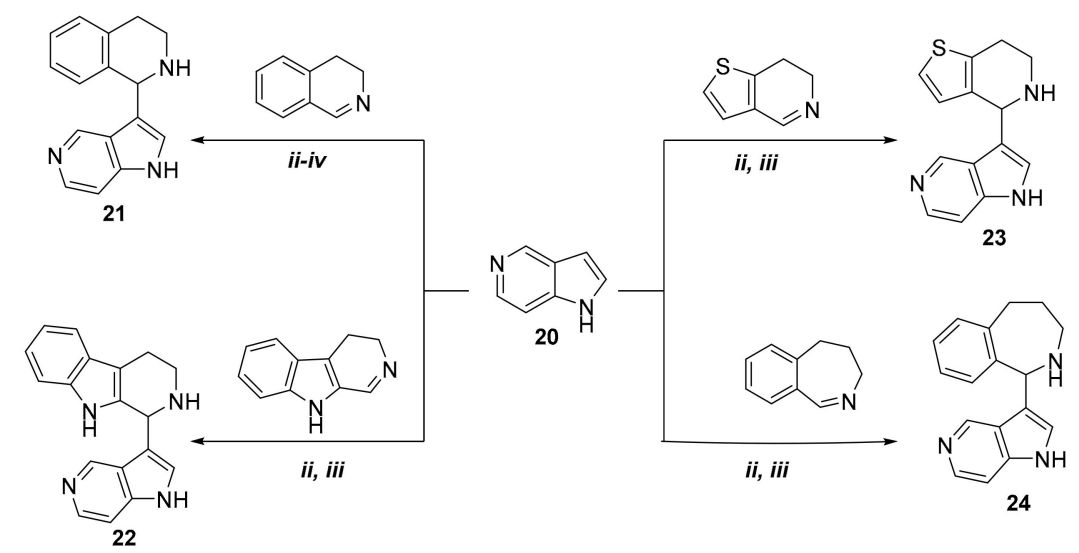

Scheme 3. Synthesis of compounds 21-24 from 5-azaindole.

Table 3. Reaction conditions for the synthesis of the products 21-24.

\begin{tabular}{ccccccc}
\hline Products & Reaction Time $^{\mathbf{a}}$ & Temperature $^{\mathbf{a}}$ & Yield $^{\mathbf{a}} \mathbf{( \% )}$ & Reaction Time $^{\mathbf{b}}$ & Temperature $^{\mathbf{b}}$ & Yield $^{\mathbf{b}}(\mathbf{\%})$ \\
\hline $\mathbf{2 1}$ & $24 \mathrm{~h}$ & $80^{\circ} \mathrm{C}(\mathrm{ii})$ & - & $2.5 \mathrm{~h}$ & $80^{\circ} \mathrm{C}(\mathrm{ii})$ & - \\
& $21 \mathrm{~h}$ & $100^{\circ} \mathrm{C}(\mathrm{iii})$ & - & $2.5 \mathrm{~h}$ & $100{ }^{\circ} \mathrm{C}(\mathrm{iii})$ & - \\
$\mathbf{2 2}$ & $8 \mathrm{~h}$ & $120^{\circ} \mathrm{C}(\mathrm{iv})$ & - & $2 \mathrm{~h}$ & $120^{\circ} \mathrm{C}(\mathrm{iv})$ & - \\
$\mathbf{2 3}$ & $19 \mathrm{~h}^{\mathrm{c}}$ & $80^{\circ} \mathrm{C}(\mathrm{ii})$ & 30 & $2.5 \mathrm{~h}^{\mathrm{c}}$ & $100^{\circ} \mathrm{C}(\mathrm{iii})$ & 72 \\
$\mathbf{2 4}$ & $15 \mathrm{~h}^{\mathrm{c}}$ & $80^{\circ} \mathrm{C}(\mathrm{ii})$ & 44 & $2 \mathrm{~h}^{\mathrm{c}}$ & $100^{\circ} \mathrm{C}(\mathrm{iii})$ & 69 \\
\hline
\end{tabular}

${ }^{\mathrm{a}}$ classical heating, ${ }^{\mathrm{b}}$ microwave irradiation, ${ }^{\mathrm{c}} \mathrm{p}$-TSA.

By applying $10 \mathrm{~mol} \%$ of $p$-TSA catalyst, C-3 aminoalkylation of 5-azaindole was extended with additional cyclic imines, such as 6,7-dihydrothieno[3,2-c]pyridine; 3,4-dihydro- $\beta$-carboline; and 4,5-dihydro-3H-benz[c]azepine (Scheme 3). Reaction conditions and yields are summarized in Table 3. As the data show, there is a difference between the reactivity of 7-, 6-, and 4-azaindoles and that of 5-azaindole-that is, for C-3 aminoalkylation of 5-azaindole an acid catalyst was needed. The lower reactivity of 5-azaindole can be accounted for by its higher pKa value (8.42), compared with those of 6-azaindole (5.61), 4-azaindole (4.85), and 7-azaindole (3.67). The given pKa values were calculated by using the Marvin Sketch software (version 16.12.12.0, calculation module developed by ChemAxon) [26]. The pKa values of azaindoles depend on the resonance stabilization of their protonated forms. The reason why the aza-Friedel-Crafts reaction of 5-azaindole occurs only under acidic conditions is that 5-azaindole has the highest basicity in the series of 4-, 5, 6-, and 7-azaindoles. It is interesting to note that the fastest reactions were achieved with the electron-rich aromatic compound 7-azaindole. This is in complete agreement with its calculated pKa value (3.67), indicating that it is the most acidic compound among the azaindoles studied.

\section{Materials and Methods}

\subsection{General Methods}

Melting points were determined on a Hinotek X-4 (Hinotek Technology Co., Ltd., Ningbo, China) melting point apparatus. Elemental analyses were performed with a Perkin-Elmer 2400 CHNS 
elemental analyzer (Perkin-Elmer, Waltham, MA, USA) in the Institute of Pharmaceutical Chemistry, University of Szeged. Merck Kieselgel 60F254 plates (Merck Hungary, Budapest, Hungary) were used for the TLC. The microwave reactions were performed with a CEM Discover SP microwave reactor (CEM, Matthwes, NC, USA).

The starting cyclic imines 3,4-dihydroisoquinoline (2) [18]; 3,4-dihydro- $\beta$-carboline (4) [19]; 6,7-dihydrothieno[3,2-c]pyridine (5) [20]; and 4,5-dihydro-3H-benz[c]azepine (6) [21,22] were synthesized according to the literature.

The ${ }^{1} \mathrm{H}$-and ${ }^{13} \mathrm{C}$-NMR spectra were recorded in $\mathrm{CDCl}_{3}$ or $\left[\mathrm{D}_{6}\right] \mathrm{DMSO}$ solution in $5 \mathrm{~mm}$ tubes at room temperature, on a Bruker Avance II spectrometer (Bruker, Karlsruhe, Germany) at $500\left({ }^{1} \mathrm{H}\right)$ and $125\left({ }^{13} \mathrm{C}\right) \mathrm{MHz}$, with the deuterium signal of the solvent as the lock and TMS as the internal standard. All spectra $\left({ }^{1} \mathrm{H},{ }^{13} \mathrm{C}\right)$ were acquired and processed with the standard BRUKER software.

Procedures

For method $\mathrm{A}$, the cyclic imine 3,4-dihydroisoquinoline $(66 \mathrm{mg}, 0.50 \mathrm{mmol})$; 6,7-dihydrothieno[3,2-c]pyridine ( $51 \mathrm{mg}, 0.38 \mathrm{mmol}$ ); 3,4-dihydro- $\beta$-carboline ( $63 \mathrm{mg}, 0.38 \mathrm{mmol}$ ) or 4,5-dihydro- $3 \mathrm{H}$-benz[c]azepine $(55 \mathrm{mg}, 0.38 \mathrm{mmol})$; and the azaindoles $(7-, 6$ - or 4 -azaindole, $(30 \mathrm{mg}$, $0.25 \mathrm{mmol})$ ) were mixed in a $25 \mathrm{~mL}$ round bottom flask. The mixture was heated in an oil bath. Reaction conditions are shown in Tables 1-3. In the case of 5-azaindole, $10 \mathrm{~mol} \%$ ( $4.3 \mathrm{mg}, 0.025 \mathrm{mmol})$ of $p$-TSA as a catalyst was also added.

For method B, the mixture of the cyclic imine 3,4-dihydroisoquinoline $(66 \mathrm{mg}, 0.50 \mathrm{mmol})$; 6,7-dihydrothieno[3,2-c]pyridine ( $51 \mathrm{mg}, 0.38 \mathrm{mmol})$; 3,4-dihydro- $\beta$-carboline $(63 \mathrm{mg}, 0.38 \mathrm{mmol}$ ) or 4,5-dihydro-3H-benz[c]azepine $(55.3 \mathrm{mg}, 0.38 \mathrm{mmol})$; and the electron-rich aromatic compound (7-, 6-, $4-$, or 5 -azaindole $(30 \mathrm{mg}, 0.25 \mathrm{mmol})$ ) were placed in a $10 \mathrm{~mL}$ pressurized reaction vial and heated in a microwave reactor, under the conditions given in Tables 1-3. In the case of 5-azaindole, $10 \mathrm{~mol} \%$ of p-TSA ( $4.3 \mathrm{mg}, 0.025 \mathrm{mmol}$ ) as a catalyst was also added.

\subsection{Synthesis of New Compounds}

\subsubsection{Synthesis of 3-(1,2,3,4-Tetrahydroisoquinolin-1-yl)-7-azaindole}

Compound 3 (Figures S1 and S2) was crystallized from $\mathrm{Et}_{2} \mathrm{O}$, recrystallized from $i-\mathrm{Pr}_{2} \mathrm{O}$. It was a white solid, with mp: $178-180^{\circ} \mathrm{C}$ (Lit.: [16] mp: 172-174 $\left.{ }^{\circ} \mathrm{C}\right)$; yield: $81 \%(51 \mathrm{mg}) ;{ }^{1} \mathrm{H}-\mathrm{NMR}\left(\left[\mathrm{D}_{6}\right]\right.$ DMSO): $\delta=2.73-2.81(1 \mathrm{H}, \mathrm{m}), 2.91-3.00(2 \mathrm{H}, \mathrm{m}), 3.10-3.17(1 \mathrm{H}, \mathrm{m}), 5.29(1 \mathrm{H}, \mathrm{s}), 6.79(1 \mathrm{H}, \mathrm{d}, J=7.9 \mathrm{~Hz})$, $6.87(1 \mathrm{H}, \mathrm{t}, J=7.6 \mathrm{~Hz}), 6.88-6.93(1 \mathrm{H}, \mathrm{m}), 6.97(1 \mathrm{H}, \mathrm{t}, J=5.2 \mathrm{~Hz}), 7.07-7.16(2 \mathrm{H}, \mathrm{m}), 7.24(1 \mathrm{H}, \mathrm{s}), 7.65(1 \mathrm{H}$, $\mathrm{d}, J=7.7 \mathrm{~Hz}), 8.14(1 \mathrm{H}, \mathrm{d}, J=5.1 \mathrm{~Hz})$, and $11.40(1 \mathrm{H}, \mathrm{s}) ;{ }^{13} \mathrm{C}-\mathrm{NMR}\left(\left[\mathrm{D}_{6}\right] \mathrm{DMSO}\right): \delta=29.7,42.3,54.5$, $115.3,117.5,118.9,125.3,125.8,126.3,127.6,128.6,129.3,135.6,139.2,142.9$, and 149.4. The analysis calculated for $\mathrm{C}_{16} \mathrm{H}_{15} \mathrm{~N}_{3}$ found C: $77.08 ; \mathrm{H}$ : 6.06; and $\mathrm{N}$ : 16.85 ; found values were $\mathrm{C}$ : 77.10; $\mathrm{H}$ : 5.98, and N: 16.88.

\subsubsection{Synthesis of 3-(1,2,3,4-Tetrahydro- $\beta$-carboline-1-yl)-7-azaindole}

Product 7 (Figures S3 and S4) was crystallized from $\mathrm{Et}_{2} \mathrm{O}$ and recrystallized from $i$ - $\mathrm{Pr}_{2} \mathrm{O}$. It was a white solid, with mp: $219-220{ }^{\circ} \mathrm{C}$; yield: $75 \%(55 \mathrm{mg}) ;{ }^{1} \mathrm{H}-\mathrm{NMR}\left(\left[\mathrm{D}_{6}\right] \mathrm{DMSO}\right): \delta=2.64-2.73(1 \mathrm{H}$, m), 2.75-2.84 $(1 \mathrm{H}, \mathrm{m}), 2.93-3.01(1 \mathrm{H}, \mathrm{m}), 3.13-3.21(1 \mathrm{H}, \mathrm{m}), 5.38(1 \mathrm{H}, \mathrm{s}), 6.87-7.00(3 \mathrm{H}, \mathrm{m}), 7.15(1 \mathrm{H}$, $\mathrm{d}, J=7.8 \mathrm{~Hz}), 7.30(1 \mathrm{H}, \mathrm{s}), 7.42(1 \mathrm{H}, \mathrm{d}, J=7.6 \mathrm{~Hz}), 7.63(1 \mathrm{H}, \mathrm{d}, J=8.4 \mathrm{~Hz}), 8.14(1 \mathrm{H}, \mathrm{d}, J=4.4 \mathrm{~Hz})$, $10.38(1 \mathrm{H}, \mathrm{s}$,$) , and 11.50(1 \mathrm{H}, \mathrm{s}, \mathrm{brs}) ;{ }^{13} \mathrm{C}-\mathrm{NMR}\left(\left[\mathrm{D}_{6}\right] \mathrm{DMSO}\right): \delta=22.0,42.1,49.7,107.4,107.9,111.6$, $118.1,118.8,121.3,123.8,126.6,127.2,135.0,136.4,140.3,140.4$, and 143.1. The analysis calculated for $\mathrm{C}_{18} \mathrm{H}_{16} \mathrm{~N}_{4}$ found $\mathrm{C}: 74.98, \mathrm{H}: 5.59$, and N: 19.43; the found values were $\mathrm{C}: 74.91, \mathrm{H}: 5.60$, and N: 19.45 .

\subsubsection{Synthesis of 3-(4,5,6,7-Tetrahydrothieno[3,2-c]pyridin-4-yl)-7-azaindole}

Product 8 (Figures S5 and S6) was crystallized from $\mathrm{Et}_{2} \mathrm{O}$ and recrystallized from $i$ - $\mathrm{Pr}_{2} \mathrm{O}$. It was a white solid, with mp: $191-195{ }^{\circ} \mathrm{C}$; yield: $89 \%(58 \mathrm{mg}) ;{ }^{1} \mathrm{H}-\mathrm{NMR}\left(\left[\mathrm{D}_{6}\right] \mathrm{DMSO}\right): \delta=2.74-2.81(1 \mathrm{H}, \mathrm{m})$, 
2.85-3.01 (2H, m), 3.12-3.20 (1H, m), $5.25(1 \mathrm{H}, \mathrm{s}), 6.49(1 \mathrm{H}, \mathrm{d}, J=6.1 \mathrm{~Hz}), 6.92-6.98(1 \mathrm{H}, \mathrm{m}), 7.14(1 \mathrm{H}, \mathrm{d}$, $J=5.3 \mathrm{~Hz}), 7.20-7.24(1 \mathrm{H}, \mathrm{m}), 7.71(1 \mathrm{H}, \mathrm{d}, J=9.6 \mathrm{~Hz}), 8.15(1 \mathrm{H}, \mathrm{d}, J=7.2 \mathrm{~Hz}), 7.42(1 \mathrm{H}, \mathrm{d}, J=7.9 \mathrm{~Hz})$, and $11.14(1 \mathrm{H}, \mathrm{s},) ;{ }^{13} \mathrm{C}-\mathrm{NMR}\left(\mathrm{CDCl}_{3}\right): \delta=26.1,42.2,52.3,115.8,117.0,119.0,121.8,123.8,126.4,128.2$, 134.7, 136.6, 143.0, and 149.1 The analysis calculated for $\mathrm{C}_{14} \mathrm{H}_{13} \mathrm{~N}_{3} \mathrm{~S}$ was $\mathrm{C}: 65.85, \mathrm{H}: 5.13$, and N: 16.46 ; found values were C: $65.83, \mathrm{H}: 5.15$, and $\mathrm{N}: 16.50$.

\subsubsection{Synthesis of 3-(2,3,4,5-Tetrahydro-1H-benz[c]azepin-1-yl)-7-azaindole}

Product 9 (Figures S7 and S8)was crystallized from $\mathrm{Et}_{2} \mathrm{O}$ and recrystallized from $i-\mathrm{Pr}_{2} \mathrm{O}$. It was a white solid, with mp: $192-194{ }^{\circ} \mathrm{C}$; yield: $78 \%(52 \mathrm{mg}) ;{ }^{1} \mathrm{H}-\mathrm{NMR}\left(\left[\mathrm{D}_{6}\right] \mathrm{DMSO}\right): \delta=2.87-2.96(1 \mathrm{H}, \mathrm{m})$, 2.97-3.14 (3H, m), $3.30(2 \mathrm{H}, \mathrm{s}), 5.40(1 \mathrm{H}, \mathrm{s}), 6.80(1 \mathrm{H}, \mathrm{d}, J=8.1 \mathrm{~Hz}), 6.95-7.07(3 \mathrm{H}, \mathrm{m}), 7.11(1 \mathrm{H}, \mathrm{t}, J=7.2$ $\mathrm{Hz}), 7.19(1 \mathrm{H}, \mathrm{d}, J=7.4 \mathrm{~Hz}), 7.84(1 \mathrm{H}, \mathrm{d}, J=8.4 \mathrm{~Hz}), 8.19(1 \mathrm{H}, \mathrm{d}, J=5.5 \mathrm{~Hz})$, and $11.45(1 \mathrm{H}, \mathrm{s}) ;{ }^{13} \mathrm{C}-\mathrm{NMR}$ ([D $\left.\left.\mathrm{D}_{6}\right] \mathrm{DMSO}\right): \delta=29.9,35.5,49.6,59.7,115.2,115.5,119.1,124.5,126.1,127.1,128.0,128.6,130.1,142.5$, $143.0,144.8,149.8$, The analysis calculated for $\mathrm{C}_{17} \mathrm{H}_{17} \mathrm{~N}_{3}$ was $\mathrm{C}: 77.54, \mathrm{H}: 6.51$, and N: 15.96; found values were C: $77.60, \mathrm{H}: 6.52$, and $\mathrm{N}: 15.98$.

\subsubsection{Synthesis of 3-(1,2,3,4-Tetrahydroisoquinolin-1-yl)-4-azaindole}

Product 12 (Figures S9 and S10) was crystallized from $\mathrm{Et}_{2} \mathrm{O}$ and recrystallized from $i$ - $\mathrm{Pr}_{2} \mathrm{O}$. It was a light beige solid, with mp: $166-168^{\circ} \mathrm{C}$; yield: $70 \%(44 \mathrm{mg}) ;{ }^{1} \mathrm{H}-\mathrm{NMR}\left(\left[\mathrm{D}_{6}\right] \mathrm{DMSO}\right): \delta=2.74-2.84(1 \mathrm{H}$, $\mathrm{m}), 2.85-2.93(2 \mathrm{H}, \mathrm{m}), 3.04-3.11(1 \mathrm{H}, \mathrm{m}), 5.50(1 \mathrm{H}, \mathrm{s}), 6.94-7.02(2 \mathrm{H}, \mathrm{m}), 7.06-7.15(4 \mathrm{H}, \mathrm{m}), 7.74(1 \mathrm{H}, \mathrm{d}$, $J=8.1 \mathrm{~Hz}), 8.32(1 \mathrm{H}, \mathrm{d}, J=4.6 \mathrm{~Hz})$, and $11.12(1 \mathrm{H}, \mathrm{s}) ;{ }^{13} \mathrm{C}-\mathrm{NMR}\left(\left[\mathrm{D}_{6}\right] \mathrm{DMSO}\right): \delta=29.7,41.4,52.6,116.8$, $119.0,119.4,125.6,126.2,127.8,127.9,129.2,129.3,135.6,139.6,142.3$, and 144.9; The analysis calculated for $\mathrm{C}_{16} \mathrm{H}_{15} \mathrm{~N}_{3}$ was $\mathrm{C}: 77.08, \mathrm{H}: 6.06$, and $\mathrm{N}: 16.85$; found values were $\mathrm{C}: 77.09, \mathrm{H}: 5.99$, and $\mathrm{N}: 16.89$.

\subsubsection{Synthesis of 3-(1,2,3,4-Tetrahydroisoquinolin-1-yl)-6-azaindole}

Product 13 (Figures S11 and S12) was purified by column chromatography (Dichloro-methane(DCM)/MeOH 9:1) and crystallized from $n$-hexane. It was a light beige solid, with mp: $152-154{ }^{\circ} \mathrm{C}$; yield: $65 \%(41 \mathrm{mg}) ;{ }^{1} \mathrm{H}-\mathrm{NMR}$ ([D $\left.\left.\mathrm{D}_{6}\right] \mathrm{DMSO}\right): \delta=2.72-2.79(1 \mathrm{H}, \mathrm{m}), 2.89-2.98$ $(2 \mathrm{H}, \mathrm{m}), 3.08-3.14(1 \mathrm{H}, \mathrm{m}), 5.29(1 \mathrm{H}, \mathrm{s}), 6.76(1 \mathrm{H}, \mathrm{d}, J=8.1 \mathrm{~Hz}), 6.96(1 \mathrm{H}, \mathrm{t}, J=5.6 \mathrm{~Hz}), 7.05-7.15(2 \mathrm{H}$, m), $7.25(1 \mathrm{H}, \mathrm{d}, J=7.5 \mathrm{~Hz}), 7.36(1 \mathrm{H}, \mathrm{s}), 7.94(1 \mathrm{H}, \mathrm{d}, J=6.1 \mathrm{~Hz}), 8.68(1 \mathrm{H}, \mathrm{s})$, and $11.36(1 \mathrm{H}, \mathrm{s}) ;{ }^{13} \mathrm{C}-\mathrm{NMR}$ ([D $\left.\left.\mathrm{D}_{6}\right] \mathrm{DMSO}\right): \delta=29.8,42.4,54.01,115.0,118.8,125.8,126.3,127.5,128.7,129.3,130.9,134.3,134.8,135.7$, 137.5, and 139.5 The analysis calculated for $\mathrm{C}_{16} \mathrm{H}_{15} \mathrm{~N}_{3}$ was $\mathrm{C}: 77.08, \mathrm{H}: 6.06$, and $\mathrm{N}: 16.85$; found values were C: $77.12, \mathrm{H}: 5.98$, and N: 16.87 .

\subsubsection{Synthesis of 3-(1,2,3,4-Tetrahydro- $\beta$-carboline-1-yl)-4-azaindole}

Product 14 (Figures S13 and S14) was crystallized from $\mathrm{Et}_{2} \mathrm{O}$, recrystallized from $i$ - $\mathrm{Pr}_{2} \mathrm{O}$. It was a light brown solid, with mp: $157-160{ }^{\circ} \mathrm{C}$; yield: $69 \%(50 \mathrm{mg}) ;{ }^{1} \mathrm{H}-\mathrm{NMR}(500 \mathrm{MHz}$, ([D 6 ]DMSO): $\delta=$ 2.86-3.06 (2H, m); 3.37-3.54 (1H, m), $6.04(1 \mathrm{H}, \mathrm{s}), 6.94-7.09(2 \mathrm{H}, \mathrm{m}), 7.14-7.29(2 \mathrm{H}, \mathrm{m}), 7.43-7.58(2 \mathrm{H}$, m), $7.85(1 \mathrm{H}, \mathrm{d}, J=8.6 \mathrm{~Hz}), 8.34-8.45(1 \mathrm{H}, \mathrm{m}), 10.81(1 \mathrm{H}, \mathrm{s}$,$) , and 11.60(1 \mathrm{H}, \mathrm{s}, \mathrm{brs}) .{ }^{13} \mathrm{C}-\mathrm{NMR}\left(\mathrm{CDCl}_{3}\right): \delta$ $=22.4,42.1,49.0,107.78,111.4,117.1,118.0,118.9,119.0,121.3,125.6,127.2,129.6,135.4,135.6,142.6$, 143.0, and 144.0. The analysis calculated for $\mathrm{C}_{18} \mathrm{H}_{16} \mathrm{~N}_{4}$ was $\mathrm{C}: 74.98, \mathrm{H}: 5.59$, and $\mathrm{N}: 19.43$; found values were C: 74.97, H: 5.62, N: 19.43.

\subsubsection{Synthesis of 3-(1,2,3,4-Tetrahydro- $\beta$-carboline-1-yl)-6-azaindole}

Product 15 (Figures S15 and S16) was purified by column chromatography (DCM/MeOH 3:1) and crystallized from $n$-hexane. It was a light brown solid, with mp: $217-219{ }^{\circ} \mathrm{C}$; yield: $62 \%(45 \mathrm{mg}) ;{ }^{1} \mathrm{H}-\mathrm{NMR}$ ([D $\left.\left.\mathrm{D}_{6}\right] \mathrm{DMSO}\right): \delta=2.65-2.84(2 \mathrm{H}, \mathrm{m}), 2.93-3.02(1 \mathrm{H}, \mathrm{m}), 3.11-3.19(1 \mathrm{H}, \mathrm{m}), 6.92-6.99(2 \mathrm{H}, \mathrm{m}), 5.43(1 \mathrm{H}, \mathrm{s}), 7.18$ $(1 \mathrm{H}, \mathrm{d}, J=7.6 \mathrm{~Hz}), 7.24(1 \mathrm{H}, \mathrm{d}, J=5.4 \mathrm{~Hz}), 7.38-4.44(1 \mathrm{H}, \mathrm{m}), 7.94(1 \mathrm{H}, \mathrm{d}, J=5.6 \mathrm{~Hz}), 8.70(1 \mathrm{H}, \mathrm{s}), 10.37(1 \mathrm{H}$, $\mathrm{s}$ ), and $11.44(1 \mathrm{H}, \mathrm{s}, \mathrm{brs}) ;{ }^{13} \mathrm{C}-\mathrm{NMR}\left(\left[\mathrm{D}_{6}\right] \mathrm{DMSO}\right): \delta=22.6,42.4,49.8,108.4,111.52,114.62,118.02,118.60$, 
$120.8,127.3,128.9,131.1,134.3,134.9,136.0,136.2$, and 137.7. The analysis calculated for $\mathrm{C}_{18} \mathrm{H}_{16} \mathrm{~N}_{4}$ was $\mathrm{C}$ : 74.98, H: 5.59, and N: 19.43; found values were C: 74.96, H: 5.61, and N: 19.44 .

\subsubsection{Synthesis of 3-(4,5,6,7-Tetrahydrothieno[3,2-c]pyridin-4-yl)-4-azaindole}

Product 16 (Figures S17 and S18) was crystallized from $\mathrm{Et}_{2} \mathrm{O}$ and recrystallized from $i-\mathrm{Pr}_{2} \mathrm{O}$. It was a white solid, with mp: $179-182{ }^{\circ} \mathrm{C}$; yield: $85 \%(55 \mathrm{mg}) ;{ }^{1} \mathrm{H}-\mathrm{NMR}\left(\left[\mathrm{D}_{6}\right] \mathrm{DMSO}\right): \delta=2.73-2.82(2 \mathrm{H}, \mathrm{m})$, 2.90-2.98 (1H, m), 3.11-3.15 (1H, m), $5.25(1 \mathrm{H}, \mathrm{s}), 6.50(1 \mathrm{H}, \mathrm{d}, J=4.9 \mathrm{~Hz}), 6.91(1 \mathrm{H}, \mathrm{t}, J=7.4 \mathrm{~Hz}), 7.05$ $(1 \mathrm{H}, \mathrm{t}, J=7.6 \mathrm{~Hz}), 7.07(1 \mathrm{H}, \mathrm{s}), 7.13(1 \mathrm{H}, \mathrm{d}, J=5.1 \mathrm{~Hz}), 7.34(1 \mathrm{H}, \mathrm{t}, J=7.9 \mathrm{~Hz}), 7.42(1 \mathrm{H}, \mathrm{d}, J=7.9 \mathrm{~Hz})$, $10.85(1 \mathrm{H}, \mathrm{s}), 8.19(1 \mathrm{H}, \mathrm{s}, \mathrm{brs}), 11.52(1 \mathrm{H}, \mathrm{s}$,$) , and 16.78(1 \mathrm{H}, \mathrm{s}, \mathrm{brs}) ;{ }^{13} \mathrm{C}-\mathrm{NMR}\left(\mathrm{CDCl}_{3}\right): \delta=25.6,41.3$, 50.6, 117.1, 118.0, 118.5, 121.9, 126.3, 126.7, 129.3, 134.7, 135.8, 143.0, and 144.4. The analysis calculated for $\mathrm{C}_{14} \mathrm{H}_{13} \mathrm{~N}_{3} \mathrm{~S}$ was $\mathrm{C}: 65.85, \mathrm{H}: 5.13, \mathrm{~N}$ : 16.46 ; found values were $\mathrm{C}: 65.82, \mathrm{H}: 5.12$, and $\mathrm{N}: 16.48$.

3.2.10. Synthesis of 3-(4,5,6,7-Tetrahydrothieno[3,2-c]pyridin-4-yl)-6-azaindole

Product 17 (Figures S19 and S20) was purified by column chromatography (DCM/MeOH 9:1) and crystallized from $n$-hexane. It was a white solid, with mp: $167-169{ }^{\circ} \mathrm{C}$; yield: $75 \%(49 \mathrm{mg}) ;{ }^{1} \mathrm{H}-\mathrm{NMR}$ ([D $\left.\left.\mathrm{D}_{6}\right] \mathrm{DMSO}\right): \delta=2.71-2.80(1 \mathrm{H}, \mathrm{m}), 2.82-2.99(2 \mathrm{H}, \mathrm{m}), 3.09-3.16(1 \mathrm{H}, \mathrm{m}), 5.23(1 \mathrm{H}, \mathrm{s}), 6.46(1 \mathrm{H}, \mathrm{d}$, $J=5.1 \mathrm{~Hz}), 7.13(1 \mathrm{H}, \mathrm{d}, J=5.6 \mathrm{~Hz}), 7.25-7.40(2 \mathrm{H}, \mathrm{m}), 7.98(1 \mathrm{H}, \mathrm{d}, J=5.4 \mathrm{~Hz}), 8.68(1 \mathrm{H}, \mathrm{s})$, and 11.35 $(1 \mathrm{H}, \mathrm{s}) ;{ }^{13} \mathrm{C}-\mathrm{NMR}\left(\mathrm{CDCl}_{3}\right): \delta=26.0,42.2,51.8,114.2,118.3,122.0,126.1,127.4,131.3,133.8,134.1,134.8$, 136.4, and 138.2. The analysis calculated for $\mathrm{C}_{14} \mathrm{H}_{13} \mathrm{~N}_{3} \mathrm{~S}$ was $\mathrm{C}: 65.85, \mathrm{H}: 5.13$, and N: 16.46; found values were $\mathrm{C}: 65.83, \mathrm{H}: 5.15$, and $\mathrm{N}: 16.45$.

\subsubsection{Synthesis of 3-(2,3,4,5-Tetrahydro-1H-benzo[c]azepin-1-yl)-4-azaindole}

Product 18 (Figures S21 and S22) was crystallized from $\mathrm{Et}_{2} \mathrm{O}$ and recrystallized from $i-\mathrm{Pr}_{2} \mathrm{O}$. It was a light beige solid, with mp: $162-165{ }^{\circ} \mathrm{C}$; yield: $68 \%(45 \mathrm{mg}) ;{ }^{1} \mathrm{H}-\mathrm{NMR}(500 \mathrm{MHz}$, ([D 6 ]DMSO): $\delta=$ 2.95-3.03 (1H, m), 3.18-3.29 (3H, m), 3.30-3.40 (2H, m), $6.06(1 \mathrm{H}, \mathrm{s}), 6.85(1 \mathrm{H}, \mathrm{d}, J=7.4 \mathrm{~Hz}), 7.06(1 \mathrm{H}, \mathrm{t}$, $J=7.9 \mathrm{~Hz}), 7.18-7.25(2 \mathrm{H}, \mathrm{m}), 7.29(1 \mathrm{H}, \mathrm{d}, J=7.4 \mathrm{~Hz}), 7.60(1 \mathrm{H}, \mathrm{s}), 7.90(1 \mathrm{H}, \mathrm{d}, J=8.4 \mathrm{~Hz}), 8.35(1 \mathrm{H}, \mathrm{d}, J$ $=4.6 \mathrm{~Hz})$, and $11.76(1 \mathrm{H}, \mathrm{s}) ;{ }^{13} \mathrm{C}-\mathrm{NMR}(100 \mathrm{MHz}$, [D6]DMSO): $\delta=25.9,34.0,49.4,57.5,111.4,117.6$, $119.8,126.6,128.7,128.8,129.3,129.4,130.0,138.0,142.5,142.9$, and 144.1. The analysis calculated for $\mathrm{C}_{17} \mathrm{H}_{17} \mathrm{~N}_{3}$ was C: $77.54, \mathrm{H}: 6.51$, and N: 15.96 ; found values were $\mathrm{C}: 77.53, \mathrm{H}: 6.53$, and N: 15.99 .

3.2.12. Synthesis of 3-(2,3,4,5-Tetrahydro-1H-benzo[c]azepin-1-yl)-6-azaindole

Product 19 (Figures S23 and S24) was purified by column chromatography (DCM/MeOH 9:1) and crystallized from $n$-hexane. It was a light beige solid, with mp: $154-156{ }^{\circ} \mathrm{C}$; yield: $64 \%(42 \mathrm{mg})$; ${ }^{1} \mathrm{H}-\mathrm{NMR}\left(500 \mathrm{MHz},\left(\left[\mathrm{D}_{6}\right] \mathrm{DMSO}\right): \delta=2.91-2.29(1 \mathrm{H}, \mathrm{m}), 3.13-3.30(2 \mathrm{H}, \mathrm{m}), 5.89(1 \mathrm{H}, \mathrm{s}), 6.86(1 \mathrm{H}, \mathrm{d}\right.$, $J=7.9 \mathrm{~Hz}), 7.08(1 \mathrm{H}, \mathrm{t}, J=7.4 \mathrm{~Hz}), 7.17-7.30(3 \mathrm{H}, \mathrm{m}), 7.48(1 \mathrm{H}, \mathrm{d}, J=5.3 \mathrm{~Hz}), 7.53(1 \mathrm{H}, \mathrm{s}), 8.07(1 \mathrm{H}, \mathrm{d}, J$ $=5.3 \mathrm{~Hz}), 8.81(1 \mathrm{H}, \mathrm{s})$, and $11.86(1 \mathrm{H}, \mathrm{s}) ;{ }^{13} \mathrm{C}-\mathrm{NMR}\left(100 \mathrm{MHz},\left[\mathrm{D}_{6}\right] \mathrm{DMSO}\right): \delta=33.8,34.3,49.0,50.1$, $50.8,58.2,114.6,126.7,126.9,128.6,128.9,129.4,129.7,130.3,130.5,131.0,134.2,135.4,138.2$, and 142.6. The analysis calculated for $\mathrm{C}_{17} \mathrm{H}_{17} \mathrm{~N}_{3}$ was $\mathrm{C}: 77.54, \mathrm{H}: 6.51$, and $\mathrm{N}$ : 15.96 ; found values were $\mathrm{C}: 77.56$, $\mathrm{H}: 6.50$, and N: 15.94 .

\subsubsection{Synthesis of 3-(1,2,3,4-Tetrahydroisoquinolin-1-yl)-5-azaindole}

Product 20 (Figures S25 and S26) was purified by column chromatography (DCM/MeOH 9:1) and crystallized from $n$-hexane. It was a light beige solid, with mp: $118-119{ }^{\circ} \mathrm{C}$; yield: $72 \%(46$ $\mathrm{mg}) ;{ }^{1} \mathrm{H}-\mathrm{NMR}\left(500 \mathrm{MHz},\left(\left[\mathrm{D}_{6}\right] \mathrm{DMSO}\right): \delta=2.88-3.39(4 \mathrm{H}, \mathrm{m}), 6.35(1 \mathrm{H}, \mathrm{s}), 6.92(1 \mathrm{H}, \mathrm{d}, J=7.8 \mathrm{~Hz})\right.$, 7.27-7.49(3H, m), 7.80-7.92 (1H, m), $8.03(1 \mathrm{H}, \mathrm{d}, J=7.1 \mathrm{~Hz}), 8.45(1 \mathrm{H}, \mathrm{d}, J=7.1 \mathrm{~Hz}), 9.14(1 \mathrm{H}, \mathrm{s})$, and $13.25(1 \mathrm{H}, \mathrm{s}) ;{ }^{13} \mathrm{C}-\mathrm{NMR}\left(100 \mathrm{MHz},\left[\mathrm{D}_{6}\right] \mathrm{DMSO}\right): \delta=29.7,42.3,54.1,107.3,118.8,123.5,125.8,125.9$, 126.4, 127.6, 129.3, 135.6, 139.3, 140.3, 140.5, and 143.6. The analysis calculated for $\mathrm{C}_{16} \mathrm{H}_{15} \mathrm{~N}_{3}$ was $\mathrm{C}$ : 77.08, H: 6.06, and N: 16.85; found values were C: 77.02, H: 5.98, and N: 16.88 . 
3.2.14. Synthesis of 3-(1,2,3,4-Tetrahydro- $\beta$-carboline-1-yl)-5-azaindole

Product 22 (Figures S27 and S28) was purified by column chromatography (DCM/MeOH 3:1) and crystallized from $n$-hexane. It was a light brown solid, with mp: $187-189{ }^{\circ} \mathrm{C}$; yield: $69 \%$ (50 $\mathrm{mg}) ;{ }^{1} \mathrm{H}-\mathrm{NMR}\left(500 \mathrm{MHz},\left(\left[\mathrm{D}_{6}\right] \mathrm{DMSO}\right): \delta=2.75-2.82(1 \mathrm{H}, \mathrm{m}), 2.84-2.91(1 \mathrm{H}, \mathrm{m}), 3.07-3.13(1 \mathrm{H}, \mathrm{m})\right.$, 3.22-3.27 $(1 \mathrm{H}, \mathrm{m}), 5.63(1 \mathrm{H}, \mathrm{s}), 6.97-7.03(2 \mathrm{H}, \mathrm{m}), 7.18(1 \mathrm{H}, \mathrm{d}, J=6.6 \mathrm{~Hz}), 7.32-7.38(2 \mathrm{H}, \mathrm{m}), 7.47(1 \mathrm{H}, \mathrm{d}$, $J=5.4 \mathrm{~Hz}), 8.11(1 \mathrm{H}, \mathrm{d}, J=4.5 \mathrm{~Hz}), 8.49(1 \mathrm{H}, \mathrm{s}),, 10.53(1 \mathrm{H}, \mathrm{s}$,$) , and 11.50(1 \mathrm{H}, \mathrm{s}) ;{ }^{13} \mathrm{C}-\mathrm{NMR}(100 \mathrm{MHz}$, [D $\mathrm{D}_{6}$ ]DMSO): $\delta=22.0,42.1,49.6,107.3,107.9,111.6,115.2,118.1,118.8,121.3,123.8,126.6,127.2,135.0$, 136.4, 140.3, 140.4, and 143.1. The analysis calculated for $\mathrm{C}_{18} \mathrm{H}_{16} \mathrm{~N}_{4}$ was $\mathrm{C}: 74.98, \mathrm{H}: 5.59$, and N: 19.43; found values were C: $74.93, \mathrm{H}: 5.58$, and N: 19.46.

\subsubsection{Synthesis of 3-(4,5,6,7-Tetrahydrothieno[3,2-c]pyridin-4-yl)-5-azaindole}

Product 23 (Figures S29 and S30) was purified by column chromatography (DCM/MeOH 9:1) and crystallized from $n$-hexane. It was a light beige solid, with $\mathrm{mp}: 113-115^{\circ} \mathrm{C}$; yield: $80 \%(52 \mathrm{mg})$; ${ }^{1} \mathrm{H}-\mathrm{NMR}\left(\left[\mathrm{D}_{6}\right] \mathrm{DMSO}\right): \delta=2.80-3.23(4 \mathrm{H}, \mathrm{m}), 5.26(1 \mathrm{H}, \mathrm{s}), 6.48(1 \mathrm{H}, \mathrm{d}, J=5.1 \mathrm{~Hz}), 6.98-7.40(3 \mathrm{H}, \mathrm{m})$, $8.08(1 \mathrm{H}, \mathrm{d}, J=5.5 \mathrm{~Hz}), 8.16(1 \mathrm{H}, \mathrm{s})$, and $11.24(1 \mathrm{H}, \mathrm{s}) ;{ }^{13} \mathrm{C}-\mathrm{NMR}\left(\left[\mathrm{D}_{6}\right] \mathrm{DMSO}\right): \delta=25.8,42.4,52.0$, $107.3,117.7,122.4,123.6,125.3,126.7,134.4,137.9,140.3,140.4$, and 143.3. The analysis calculated for $\mathrm{C}_{14} \mathrm{H}_{13} \mathrm{~N}_{3} \mathrm{~S}$ was $\mathrm{C}: 65.85, \mathrm{H}: 5.13$, and N: 16.46 ; found values were $\mathrm{C}: 65.84, \mathrm{H}: 5.14$, and $\mathrm{N}: 16.49$.

\subsubsection{Synthesis of 3-(2,3,4,5-Tetrahydro-1H-benzo[c]azepin-1-yl)-5-azaindole}

Product 24 (Figures S31 and S32) was purified by column chromatography (DCM/MeOH 9:1) and crystallized from $n$-hexane. It was a light beige solid, with mp: $142-145^{\circ} \mathrm{C}$; yield: $65 \%(43 \mathrm{mg})$; ${ }^{1} \mathrm{H}-\mathrm{NMR}\left(\left[\mathrm{D}_{6}\right] \mathrm{DMSO}\right): \delta=2.82-3.09(6 \mathrm{H}, \mathrm{m}), 5.40(1 \mathrm{H}, \mathrm{s}), 6.81(1 \mathrm{H}, \mathrm{d}, J=6.7 \mathrm{~Hz}), 6.93-7.02(1 \mathrm{H}, \mathrm{m})$, $7.07-7.14(3 \mathrm{H}, \mathrm{m}), 7.19(1 \mathrm{H}, \mathrm{d}, J=7.3 \mathrm{~Hz}), 7.35(1 \mathrm{H}, \mathrm{d}, J=7.3 \mathrm{~Hz}), 8.12(1 \mathrm{H}, \mathrm{d}, J=5.7 \mathrm{~Hz}), 8.72(1 \mathrm{H}$, s), $11.27(1 \mathrm{H}, \mathrm{brs}) ;{ }^{13} \mathrm{C}-\mathrm{NMR}\left(\mathrm{CDCl}_{3}\right): \delta=30.2,35.8,53.4,106.8,117.8,123.6,124.3,126.1,127.2,127.7$, $129.3,130.0,140.3,140.7,142.2,143.2$, and 144.0. The analysis calculated for $\mathrm{C}_{17} \mathrm{H}_{17} \mathrm{~N}_{3}$ was $\mathrm{C}: 77.54, \mathrm{H}$ : 6.51, and N: 15.96; found values were C: 77.57, H: 6.52, and N: 15.94 .

\section{Conclusions}

To conclude, the modified aza-Friedel-Crafts reaction was applied for the C-3 substitution of azaindoles. The reactions were achieved by using 3,4-dihydroisoquinoline; 6,7-dihydrothieno[3,2-c]pyridine; 3,4-dihydro- $\beta$-carboline; and 4,5-dihydro-3H-benz[c]azepine as imine substrates. The reactions of 4-azaindole and 6-azaindole with similar reactivity led to the formation of new 3-isoquinolyl-, 3-thieno[3,2-c]pyridyl-, 3- $\beta$-carbolinyl-, and 3-benz[c]azepinyl-azaindole derivatives. Starting from 5-azaindole, the modified aza-Friedel-Crafts reaction could only be performed by using $10 \mathrm{~mol} \%$ of $p$-TSA as a catalyst. Systematic correlation was found between the reactivity of azaindoles and their pKa values. This latter observation allows us to explain why the direct coupling of 5-azaindole works only under acidic conditions. Namely, 5 -azaindole $(\mathrm{pKa}=8.42)$ has the highest basicity in the series of 4-, 5-, 6-, and 7-azaindoles. It is important to note that all reactions could be accelerated by using microwave irradiation.

Supplementary Materials: Supplementary materials are available online.

Author Contributions: F.F., K.B., and I.S. planned and designed the project. I.S. and K.B. performed the syntheses and characterized the synthesized compounds. K.B., F.F., and I.S. prepared the manuscript for publication. All authors discussed the results and commented on the manuscript.

Funding: The authors thank the Ministry of National Economy, National Research Development and Innovation Office [GINOP-2.3.2-15-2016-00038], the European Union-funded Hungarian Grant [EFOP-3.6.1-16-2016-00008], and the Ministry of Human Capacities, Hungary Grant, 20391-3/2018/FEKUSTRAT.

Conflicts of Interest: The authors declare no conflict of interest. 


\section{References}

1. Ghose, A. K.; Herbertz, T.; Pippin, D. A.; Salvino, J. M.; Mallamo, J. P. Knowledge Based Prediction of Ligand Binding Modes and Rational Inhibitor Design for Kinase Grug Discovery. J. Med. Chem. 2008, 51, 5149-5155. [CrossRef] [PubMed]

2. Tang, J.; Hamajima, T.; Nakano, M.; Sato, H.; Dickerson, S.H.; Lackey, K.E. Knowledge-Based Design of 7-azaindoles as Selective B-Raf Inhibitors. Bioorg. Med. Chem. Lett. 2008, 18, 4610-4614. [CrossRef] [PubMed]

3. Mérour, J.Y.; Buron, F.; Plé, K.; Bonnet, P.; Routier, S. The Azaindole Framework in the Design of Kinase Inhibitors. Molecules 2014, 19, 19935-19979. [CrossRef] [PubMed]

4. Pires, M.J.; Poeira, D.L.; Purificacao, S.I.; Marques, M.M.B. Synthesis of Substituted 4-, 5-, 6-, and 7-Azaindoles from Aminopyridines via a Cascade C-N Cross-Coupling/Heck Reaction. Org. Lett. 2016, 18, 3250-3253. [CrossRef] [PubMed]

5. Irie, T.; Sawa, M. 7-Azaindole: A Versatile Scaffold for Developing Kinase Inhibitors. Chem. Pharm. Bull. 2018, 66, 29-36. [CrossRef] [PubMed]

6. Manning, G.; Whyte, D.B.; Martinez, R.; Hunter, T.; Sudarsanam, S. The Protein Kinase Complement of the Human Genome. Science 2002, 298, 1912-1934. [CrossRef]

7. Bollag, G.; Tsai, J.; Zhang, J.; Zhang, C.; Ibrahim, P.; Nolop, K.; Hirth, P. Vemurafenib: The First Drug Approved for BRAF-Mutant Cancer. Nature 2012, 11, 873-886. [CrossRef] [PubMed]

8. Tsai, J.; Lee, J.T.; Wang, W.; Zhang, J.; Cho, H.; Mamo, S.; Bremer, R.; Gillette, S.; Kong, J.; Haass, N.K.; et al. Discovery of a Selective Inhibitor of Oncogenic B-Raf Kinase with Potent Antimelanoma Activity. Proc. Natl. Acad. Sci. USA 2008, 105, 3041-3046. [CrossRef]

9. Ito, M.; Yamazaki, S.; Yamagami, K.; Kuno, M.; Morita, Y.; Okuma, K.; Nakamura, K.; Chida, N.; Inami, M.; Inoue, T.; et al. A Novel JAK Inhibitor, Peficitinib, Demonstrates Potent Efficacy in a Rat Adjuvant-Induced Arthritis Model. J. Pharmacol. Sci. 2017, 133, 25-33. [CrossRef]

10. Tap,W.D.; Wainberg, Z.A.; Anthony, S.P.; Ibrahim, P.N.; Zhang, C.; Healey, J.H.; Chmielowski, B.; Staddon, A.P.; Cohn, A.L.; Shapiro, G.I.; et al. Structure-Guided Blockade of CSF1R Kinase in Tenosynovial Giant-Cell Tumor. Engl. J. Med. 2015, 373, 428-437. [CrossRef]

11. Adams, N.D.; Adams, J.L.; Burgess, J.L.; Chaudhari, A.M.; Copeland, R.A.; Donatelli, C.A.; Drewry, D.H.; Fisher, K.E.; Hamajima, T.; Hardwicke, M.A.; et al. Discovery of GSK1070916, A Potent and Selective Inhibitor of Aurora B/C Kinase. J. Med. Chem. 2010, 53, 3973-4001. [CrossRef] [PubMed]

12. Tokushige, H.; Inatani, M.; Nemoto, S.; Sakaki, H.; Katayama, K.; Uehata, M.; Tanihara, H. Effects of Topical Administration of Y-39983, a Selective Rho-Associated Protein Kinase Inhibitor, on Ocular Tissues in Rabbits and Monkeys. Invest. Ophthalmol. Vis. Sci. 2007, 48, 3216-3222. [CrossRef] [PubMed]

13. Szatmári, I.; Sas, J.; Fülöp, F. C-3 Functionalization of Indole Derivatives with Isoquinolines. Curr. Org. Chem. 2016, 20, 2036-2038. [CrossRef]

14. Santos, A.; Mortinho, A.; Marques, M. Metal-Catalyzed Cross-Coupling Reactions on Azaindole Synthesis and Functionalization. Molecules 2018, 23, 2673. [CrossRef] [PubMed]

15. Ghobrial, M.; Harhammer, K.; Mihovilovic, M.D.; Schmürch, M. Facile, Solvent and Ligand Free Iron Catalyzed Direct Functionalization of N-Protected Tetrahydroisoquinolines and Isochroman. Chem. Commun. 2010, 46, 8836. [CrossRef] [PubMed]

16. Ghobrial, M.; Schmürch, M.; Mihovilovic, M.D. Direct Functionalization of (Un)protected Tetrahydroisoquinoline and Isochroman under Iron and Copper Catalysis: Two Metals, Two Mechanisms. J. Org. Chem. 2011, 76, 8781-8793. [CrossRef] [PubMed]

17. Szatmári, I.; Sas, J.; Fülöp, F. Catalyst-Free Coupling of Indole Derivatives with 3,4-dihydroisoquinoline and Related Compounds. Tetrahedron Lett. 2013, 54, 5069-5071. [CrossRef]

18. Sas, J.; Szatmári, I.; Fülöp, F. One-Pot $\alpha$-Arylation of $\beta$-Carboline with Indole and Naphthol Derivatives. Curr. Org. Synth. 2016, 13, 611-616. [CrossRef]

19. Chen, Z.; Hu, G.; Li, D.; Chen, J.; Li, Y.; Zhou, H.; Xie, Y. Synthesis and Vasodilator Effects of Rutaecarpine Analogues which Might be Involved Transient Receptor Potential Vanilloid Subfamily, Member 1 (TRPV1). Bioorg. Med. Chem. 2009, 17, 2351-2359. [CrossRef]

20. Herz, W.; Tsai, L. Sulfur Analogs of Isoquinolines. IV. The Pictet-Gams Reaction and Attempts to Prepare Analogs of Papaverine1,2. J. Chem. Soc. 1955, 77, 3529-3533. [CrossRef] 
21. Meyers, A.I.; Hutchings, R.H. The Asymmetric Synthesis of 1-alkyl-2,3,4,5-tetrahydro-benzazepines and Benzo[ $\beta]$-1-azabicyclo[5,3,1]decanes. Tetrahedron 1993, 49, 1807-1820. [CrossRef]

22. Jakubec, P.; Helliwell, M.; Dixon, D.J. Cyclic Imine Nitro-Mannich/ Lactamization Cascades: A Direct Stereoselective Synthesis of Multicyclic Piperidinone Derivatives. D. J. Org. Lett. 2008, 10, 4267-4270. [CrossRef] [PubMed]

23. Khodaei, M.M.; Khosropour, A.R.; Moghanian, H. A Simple and Efficient Procedure for the Synthesis of Amidoalkyl Naphthols by $p$-TSA in Solution or under Solvent-Free Conditions. Synlett 2006, 6, 916-920. [CrossRef]

24. Khosropour, A.R.; Khodaei, M.M.; Moghanian, H. A Facile, Simple and Convenient Method for the Synthesis of 14-Alkyl or Aryl-14-H-Dibenzo[a,j]xanthenes Catalyzed by $p$ TSA in Solution and Solvent-Free Conditions. Synlett 2005, 6, 955-958. [CrossRef]

25. Csütörtöki, R.; Szatmári, I.; Mándi, A.; Kurtán, T.; Fülöp, F. Synthesis of Hydroxynaphthyl-Substituted $\alpha$-Amino Acid Derivatives via a Modified Mannich Reaction. Synlett 2011, 13, 1940-1946.

26. Marvin was used for calculating pKa values of azaindoles, Marvin 16.12.12.0, ChemAxon. 2016. Available online: http://www.chemaxon.com.

Sample Availability: Samples of the compounds are not available from the authors. 